\title{
Serious Gastrointestinal Form of Henoch-Schonlein Purpura Induced by Helicobacter pylori Infection Complicated with Bradycardia and Euthyroid Sick Syndrome
}

G Vijatov-Djuric $^{1,2}$, N Barisic ${ }^{1,2}$, A Djuretic ${ }^{2}$, D Katanic ${ }^{1,2}$, M Stojsic ${ }^{2}$, B Milanovic ${ }^{1,2}$

\begin{abstract}
Association between Henoch-Schonlein purpura and Helicobacter pylori infection is rarely reported in the literature. We present a case of Henoch-Schonlein purpura with severe gastrointestinal manifestations, bradycardia and euthyroid sick syndrome, were resolution of disease occurred only after the eradication of Helicobacter pylori infection.
\end{abstract}

Keywords: Bradycardia, euthyroid sick syndrome, Helicobacter pylori, purpura

From: ${ }^{1}$ Faculty of Medicine, University of Novi Sad, Novi Sad, Serbia. ${ }^{2}$ Institute for child and youth healthcare of Vojvodina, Novi Sad, Serbia.

Correspondence: Dr N Barisic, Department of Paediatrics, Faculty of Medicine, University of Novi Sad, Novi Sad, Hajduk Veljkova 3, 21000 Novi Sad, Serbia.

E-mail: nenad.barisic@mf.uns.ac.rs 


\section{INTRODUCTION}

Henoch-Schönlein purpura (HSP) is the most common systemic vasculitis in children ( 10 - 30 cases per 100,000 children under 17 years) and usually occurs in children younger than 10 years (1). HSP is non-granulomatous, small-vessel vasculitis, which presents with palpable purpura on the lower extremities and buttocks with possible joint, gastrointestinal and renal involvement $^{1}$. In $75 \%$ of patients, an infection precedes HSP (2). Helicobacter pylori (HP) infection is rarely associated with HSP.

We present a case of HSP with severe gastrointestinal manifestations, bradycardia and euthyroid sick syndrome, were resolution of disease occurred after the eradication of Helicobacter pylori infection.

\section{CASE REPORT}

A five-year old boy was hospitalized due to palpable purpura, abdominal pain and vomiting. The disease began four days earlier with petechial rash on the legs, followed by episodes of abdominal pain and vomiting. He was previously healthy.

On admission, he had palpable purpura on the legs and gluteus, and feet oedema. Abdomen was slightly meteoristic and sensitive to in paraumbilical region. Other findings were normal. C-reactive protein $24.3 \mathrm{mg} / \mathrm{L}(0-5 \mathrm{mg} / \mathrm{L})$, ESR $10 \mathrm{~mm} / \mathrm{h}$, WBC $20.5 \times 10^{9} / \mathrm{L}(78 \%$ granulocytes, 16\% lymphocytes ), ERC 5.49x10 $12 / \mathrm{L}$, HGB $133 \mathrm{~g} / \mathrm{L}$, HTC 43\%, PLT $305 \times 10^{9} /$ L. Coagulation profile, electrolytes, renal and liver function, immunoglobulins (Ig) A, $\mathrm{M}$ and $\mathrm{G}$, complement components $\mathrm{C} 3$ and $\mathrm{C} 4$, Antistreptolysin $\mathrm{O}$ titre: normal. Viral serology: negative. Antinuclear antibodies (ANA), lupus anticoagulant, anticardiolipin antibodies (ACLA) IgG and IgM, Anti 32 glycoproteins IgM and IgG: negative. Urine: proteinuria 2+, 15 
erythrocytes, $24-\mathrm{h}$ proteinuria: $0.154 \mathrm{~g} /$ day. Throat swab: negative. Occult blood in the stool: positive. Coproculture and Clostridium difficile toxin: negative. Ultrasonography of the abdomen showed oedematous walls of the distal part of the terminal ileum (wall thickness 5.3 $\mathrm{mm}$ ) with intense regional blood flow (Power Doppler); moderate amount of free fluid in the abdomen (Figure 1.).

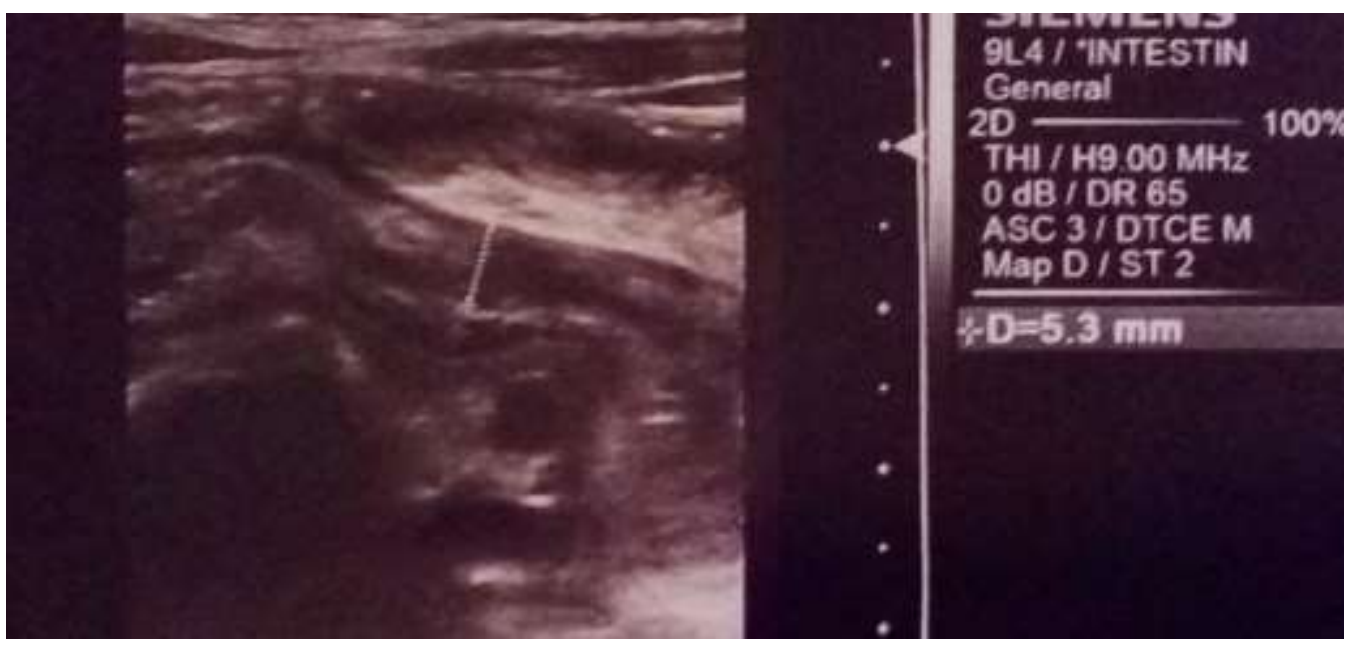

Fig. 1: Ultrasonography of the abdomen showing oedematous walls of the distal part of the terminal ileum

Our diagnosis was HSP, so we started the therapy with methylprednisolone ( $1 \mathrm{mg} / \mathrm{kg} / \mathrm{day})$ and proton pump inhibitor. On the $3^{\text {rd }}$ day, palpable purpura re-emerged, now affecting all four extremities. This was accompanied by intense abdominal pain, colicae, diarrhoea and haematochezia. Ultrasound examination of the abdomen showed advanced thickening of intestinal walls. Due to exacerbation of the disease, we administered methylprednisolone pulse therapy (30 mg/kg/day) during 3 days, and then proceed with methylprednisolone at standard dose of $1 \mathrm{mg} / \mathrm{kg} /$ day. Oral intake has been suspended and total parenteral nutrition was initiated. All these therapeutic measures gave only a short-term effect. 
On the $9^{\text {th }}$ day, previous symptoms relapsed. Ultrasonography revealed further thickening of the intestinal walls. Intravenous immunoglobulins (IVIG) $2 \mathrm{~g} / \mathrm{kg}$, were given. This was followed by significant clinical improvement, but, in attempts to reduce the doses of corticosteroids, abdominal pain and skin lesions relapsed.

During the $3^{\text {rd }}$ week of illness, the boy become bradycardic (heart rate $44 / \mathrm{min}$ ) and constipated.

Standard ECG showed sinus bradycardia. 24h ECG Holter monitoring showed persistent bradycardia with mean heart rate of 55/min, minimal heart rate of $39 / \mathrm{min}$, without significant sinus pauses and conduction disorders (Figure 2). Echocardiography was normal.

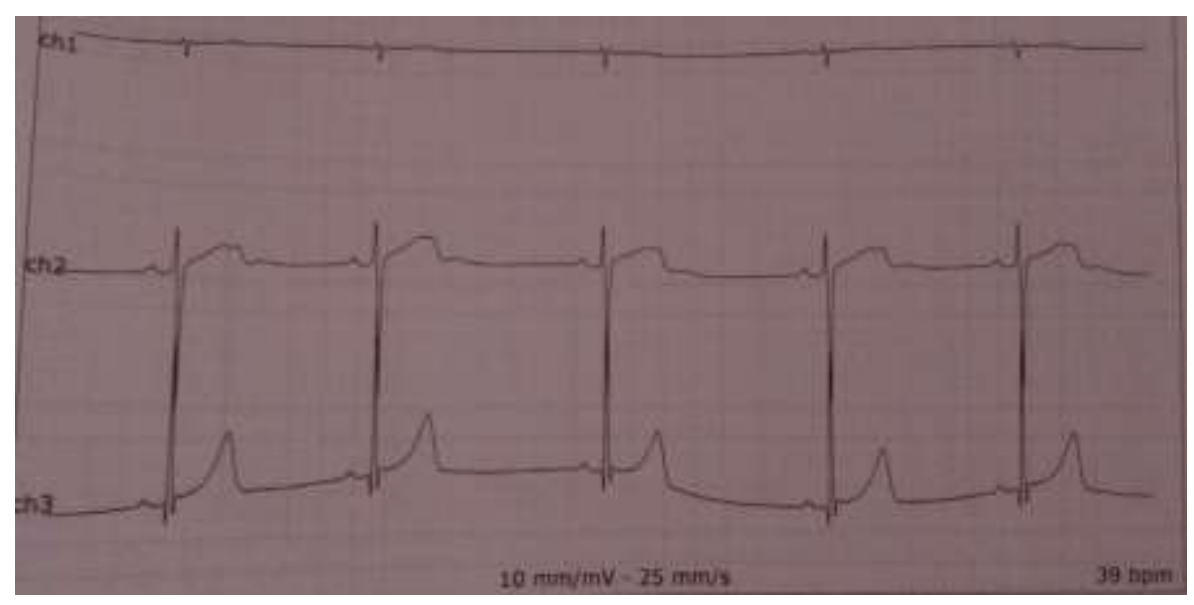

Fig 2. 24h ECG Holter monitoring showing bradycardia with heart rate of 39/min.

Thyroid function tests were indicative for euthyroid sick syndrome (triiodothyronine (T3) $0.72 \mathrm{nmol} / \mathrm{L}$ (ref. range 1.42 to $3.8 \mathrm{nmol} / \mathrm{L}$ ), normal levels of thyroxine (T4) and thyroid stimulating hormone (TSH)). Levothyroxine was introduced in the therapy and gradual normalization of the heart rate and hormones levels occurred. 
Due to relapsing gastrointestinal symptoms, anti-Helicobacter pylori IgG and IgA antibodies were determined and the results were positive for $\mathrm{IgG}$ antibodies $(90.1 \mathrm{RJ} / \mathrm{ml}$, ref. $<22 \mathrm{RJ} / \mathrm{ml}$ ), so 7-days course of amoxicillin and metronidazole has been conducted. After eradication of HP, there was no further reoccurrence of abdominal pain, findings of occult blood in the stool become and remained negative, abdominal ultrasound findings normalized, and skin lesions permanently resolved. The corticosteroids were gradually reduced and suspended after 2 months. Levothyroxine therapy has been suspended after one month.

\section{DISCUSSION}

Gastrointestinal symptoms occur in $51-74 \%$ of children with HSP and usually manifest as diffuse abdominal pain or colicae, associated with nausea, vomiting and diarrhoea $(3,4)$. There is no consensus for the treatment of severe gastrointestinal manifestations of HSP and recommendations are based on small studies and case reports. The most commonly administered therapy are methylprednisolone pulses and IVIG, and sporadically plasmapheresis, cyclophosphamide, methotrexate, mycophenolate mofetil, azathioprine, rituximab, factor XIII concentrate replacement therapy, etc. $(5,6)$ In our patient, relapsing episodes of gastrointestinal symptoms, required complete cessation of oral intake and introduction of parenteral nutrition.

Several smaller studies and case reports that indicate association between HSP and HP infection were recently published. In those cases, eradication of HP led to a prompt resolution HSP symptoms (7). In children, HP infections are asymptomatic, as was the case with our patient. Increased IgA levels, decreased C3 levels, increased cryoglobulins, and elevated levels of pro-inflammatory mediators caused by HP infection may have some role in the course of HSP (8). Previous studies indicate that the eradication of HP may lead to resolution and 
decreased recurrence of HSP, but still, there is not enough evidence to substantiate the claim that HP infection may trigger HSP (8). In our case, resolution of all symptoms after eradication of HP, suggest an association between HP infection and HSP.

In our patient, the course of disease was complicated with persistent bradycardia. There are few described cases of adult patients treated with methylprednisolone, in whom bradycardia emerged within a few hours to several days after the start of the therapy. Also, in patients treated with conventional lower doses of corticosteroids, episodes of bradycardia were reported, but, extremely rarely (9). The mechanisms by which corticosteroids may cause bradycardia have not been fully elucidated, but there are few speculations, such are that corticosteroids directly affect cardiomyocytes modulating their sensitivity to catecholamines, or cause rapid electrolyte shifts across cell membranes, or indirectly - by inducing arterial hypertension. But, in our patient, likely cause of bradycardia were decreased T3 levels. Possible mechanisms of decreased T3 availability may be corticosteroid-induced block of peripheral conversion of $\mathrm{T} 4$ to $\mathrm{T} 3$ or competition between corticosteroids and thyroid hormones for albumin binding sites what decreases T3 levels despite normal T4 levels (10).

\section{AUTHORS' NOTE}

All the authors of the manuscript have participated in the concept and design, analysis, drafting and revising of the manuscript. Each author listed on the manuscript has seen and approved the submission of this version of the manuscript and takes full responsibility for the manuscript. The authors of this paper have no any potential conflict of interest. 


\section{REFERENCES}

1. Trnka P. Henoch-Schönlein purpura in children. J Paediatr Child Health 2013; 49: 9951003.

2. Rigante D, Castellazzi L, Bosco A, Esposito S. Is there a crossroad between infections, genetics, and Henoch-Schönlein purpura? Autoimmun Rev 2013; 12: 1016-21.

3. Ebert EC. Gastrointestinal manifestations of Henoch-Schönlein purpura. Dig Dis Sci 2008; 53: 2011-9.

4. McCarthy HJ, Tizard EJ.Diagnosis and management of Henoch-Schönlein purpura. Eur J Pediatr 2010; 169: 643-50.

5. Kang HS, Chung HS, Kang KS, Han KH. High-dose methylprednisolone pulse therapy for treatment of refractory intestinal involvement caused by Henoch-Schönlein purpura: a case report. J Med Case Rep 2015; 9: 65.

6. Cheqaoui B, Chausset A, Stephan JL, Merlin E. Intravenous immunoglobulins for severe gastrointestinal involvement in pediatric Henoch-Schönlein purpura: A French retrospective study. Arch Pediatr 2016; 23: 584-90.

7. Ulas T, Tursun I, Dal MS, Eren MA, Buyukhatipoglu H. Rapid improvement of Henoch-Schönlein purpura associated with the treatment of Helicobacter pylori infection. J Res Med Sci 2012; 17: 1086-8.

8. Xiong JL, Mao M. Current views of the relationscip between Helicobacter pylori and Henoch-Schönlein purpura in children. World J Clin Pediatr 2016; 5: 82-8.

9. Taylo MR, Gaco D. Symptomatic sinus bradycardia after a treatment course of highdose oral prednisone. J Emerg Med 2013; 45: e55-8.

10. Kurtdede A, Asti RN, Sel T, Kurtdede N, Karagul H, Atalay O et al. Effects of antiinflammatory and immunosuppressive doses of prednisolone on serum 
Henoch-Schonlein Purpura Induced by Helicobacter pylori

triiodothyronine, thyroxine, and free thyroxine concentrations and thyroid morphology in the dog. Revue Med Vet 2004; 155: 324-30. 